\title{
Práticas Educativas para Autocuidado Pós-Operatório Divulgadas por Meio de Mídia Digital
}

\section{Lays Tamara Dantas-Silva ${ }^{1}$, Vanessa Carla do Nascimento Gomes Brito ${ }^{1}$, Laryssa Felinto de Araújo ${ }^{1}$, Jaqueline Queiroz de Macedo}

\author{
${ }^{1}$ Universidade Federal da Paraíba (UFPB) \\ Caixa-postal: 5045 - 58.051-970 - João Pessoa - PB - Brasil \\ ${ }^{2}$ Departamento de Enfermagem Clínica \\ Universidade Federal da Paraíba (UFPB) - João Pessoa, PB - Brasil \\ lays.tamarads@gmail.com, vanessacarlabrito@gmail.com, \\ lalafelinto@gmail.com, jaquelineqmac@gmail.com
}

\begin{abstract}
With the rapid technological development and its widespread, information technologies and communication (ITCs) can be used in innovative ways to achieve social impact, such as health education, through the dissemination of videos, texts, articles, on websites and networks social. The objective is to report the experience of conducting virtual educational actions on self-care in the postoperative period through the social network Instagram.
\end{abstract}

Resumo. Com o rápido desenvolvimento tecnológico e sua grande propagação, as tecnologias de informação e comunicação (TICs) podem ser usadas de maneiras inovadoras para obter impacto social, como a educação em saúde, através da divulgação de vídeos, textos, artigos, em websites e redes sociais. $O$ objetivo é relatar a experiência de condução de ações educativas virtuais sobre autocuidado no pós-operatório por meio da rede social Instagram.

\section{Introdução}

A educação em saúde é um instrumento que possibilita a promoção da qualidade de vida de sujeitos, família e comunidade a partir de conhecimentos técnicos e científicos fornecidos pelos profissionais e acadêmicos da área da saúde. Desta forma, contribui para a construção da consciência crítica da população sobre cuidados de saúde [Tavares et al 2018, Vieira et al 2017, Oliveira et al 2018].

Dentre as estratégias de educação em saúde, comumente são utilizadas a comunicação oral [Santos 2018], uso de materiais escritos, como folders [Massara et al 2016], vídeos [Dalmolin et al 2016]. Contudo, a Revolução Digital está modificando o modo como a sociedade se relaciona e desenvolve seus processos, o que afeta, invariavelmente a saúde, tal como apontado pelo Global Information Technology Report de 2016, elaborado por meio do World Economic Forum, o qual aponta que as tecnologias de informação e comunicação (TICs) podem ser usadas de maneiras inovadoras para obter impacto social, como atenção em saúde [Baller, Dutta e Lanvin 2016]. 
Com o rápido desenvolvimento tecnológico e sua grande propagação, a educação em saúde passou a ser realizada também de forma virtual, por meio de vídeos, textos, artigos, redes sociais e entre muitos outros, visto que esses recursos tecnológicos estão cada vez mais presentes no cotidiano das pessoas, passando a ser um excelente meio de divulgação de informações [Neto et al 2019, Aguiar et al 2018].

Cada vez mais difundida através das mídias digitais, a educação em saúde possibilita a realização da prática de autocuidado por parte dos indivíduos por meio das informações fornecidas. Por autocuidado, entende-se o estímulo para o indivíduo cuidar de si de forma consciente e efetiva, conforme orientado pela Teoria de Orem [Costa et al 2018, Queirós, Vidinha e Filho 2014].

A educação em saúde orientada para o autocuidado permite ao indivíduo ser protagonista dos cuidados necessários a serem realizados, em especial quando em seu domicílio [Santos et al 2015, Souza et al 2017].

Aliado ao uso das TICs, a propagação do uso de smartphones, apps e internet por todas as classes sociais, propicia o compartilhamento de informações por meio de mídias sociais tais como o Instagram, possibilitando assistência ao paciente, monitoramento, reabilitação, comunicação, diagnóstico, ensino e pesquisa [Boulos, Giustini e Wheeler 2016]. Utilizando a publicação de fotos e mensagens em páginas institucionais, profissionais, públicas ou privadas, este é um aplicativo gratuito disponível para as plataformas Android e iOS que pode ser acessado a partir de qualquer navegador da Web em computadores.

Nesse contexto, o presente estudo tem como objetivo relatar a experiência de condução de ações educativas virtuais sobre autocuidado no pós-operatório por meio da rede social Instagram.

\section{Sobre o uso das mídias sociais na divulgação de projeto de extensão}

Mídias sociais são definidas como a socialização e a comunicação em rede de grupos com interesses comuns através de processos contínuos de interação no ambiente digital, mediados a partir do uso da internet e de dispositivos tecnológicos, móveis ou não, como computadores, tablets e smartphones [Bissolotti et al 2014, Colnago 2015].

Atualmente, as mídias sociais integram, quase indissociavelmente, o cotidiano das pessoas e das empresas, estimulando o desenvolvimento de discussões, ditando comportamentos e tendências, e proporcionando a cadeia de valor de produtos e serviços [Colnago 2015].

Dentre as opções de mídias sociais disponíveis, o Instagram classifica-se tanto como uma rede de compartilhamento de fotos e vídeos, quanto como uma rede de cunho social, o que mostra a versatilidade do Instagram, inclusive no que diz respeito a sua utilização para fins de educação em saúde [Colnago 2015, Melo et al 2020].

O uso de mídia social como ferramenta para a educação em saúde proporciona o acesso às informações científicas, antes adquirido apenas em serviços de saúde, devido a disponibilidade da tecnologia, favorecendo a comunicação entre os profissionais de saúde e o público em geral, e contribuindo para a qualidade do autocuidado e empoderamento do público [Chaves et al 2018, Sá et al 2018, Sarwer-Foner 2018]. Vale salientar que tais ferramentas devem ser usadas com cautela, havendo sempre 
embasamento científico, para segurança dos consumidores do conteúdo [Melo et al 2020, Thomas e Fontana 2019].

No entanto, para que a informação chegue ao máximo de pessoas possível, é importante conhecer o funcionamento do algoritmo do Instagram e utilizar essa ferramenta da melhor forma.

Desde março de 2016, o Instagram (lançado em 2010) passou a utilizar um algoritmo que classifica automaticamente o que é relevante ou não para os usuários. A ideia é otimizar a experiência do usuário dentro da rede social, filtrando as publicações para que elas apareçam de acordo com seus interesses dentro do feed. Esse filtro é feito com base em três variáveis: temporalidade (priorizando as publicações mais recentes, embora não necessariamente em ordem cronológica), engajamento (interações que a postagem recebeu, como curtidas e comentários; ou seja, quanto mais interação, mais a postagem é entregue) e relacionamento (as publicações que mais vão aparecer para o usuário serão relacionadas aos perfis que ele mais interage) [Freitas, Borges e Rios 2016, Porto, 2018].

Entretanto, essa personalização do Instagram também tem seu lado negativo para o usuário, uma vez que a seletividade da rede social impossibilita o acesso aos conteúdos que não foram incluídos na seleção dos conteúdos de interesse do usuário [Freitas, Borges e Rios 2016, Porto 2018].

Assim, para que o Instagram sirva como uma boa ferramenta de educação em saúde e divulgação científica, as postagens precisam - além de linguagem acessível e conteúdo interessante - de bastante divulgação e interação por parte do público.

\section{Organização das publicações na página do projeto}

As publicações são divulgadas em um perfil, na rede social Instagram, elaborado exclusivamente para propagar as ações do projeto (@autocuidado_poscirurgia). As informações publicadas referem-se às orientações gerais acerca do autocuidado pósoperatório e orientações específicas relacionadas a diferentes tipos de cirurgias. Tais postagens, na perspectiva das ações de educação em saúde, objetivam levar conhecimento e autonomia para o público-alvo, através de linguagem acessível, porém com embasamento científico.

O conteúdo das publicações segue um padrão. São utilizadas imagens atrativas, criadas exclusivamente para o perfil@autocuidado_poscirurgia, acompanhadas de legendas que expõem o tema de forma breve e clara, seguido de hashtags relacionadas ao tema e à instituição sede do projeto.

Os primeiros conteúdos produzidos pelo projeto foram referentes aos conhecimentos gerais acerca de procedimentos cirúrgicos: a) começando com um passoa-passo para a realização correta da limpeza da ferida cirúrgica, b) seguido da explanação acerca da alimentação e hidratação durante o período pós-operatório, c) o retorno às atividades de vida diária após a cirurgia, d) e uma postagem desvendando mitos comuns acerca do período pós-operatório (Figura 1). 

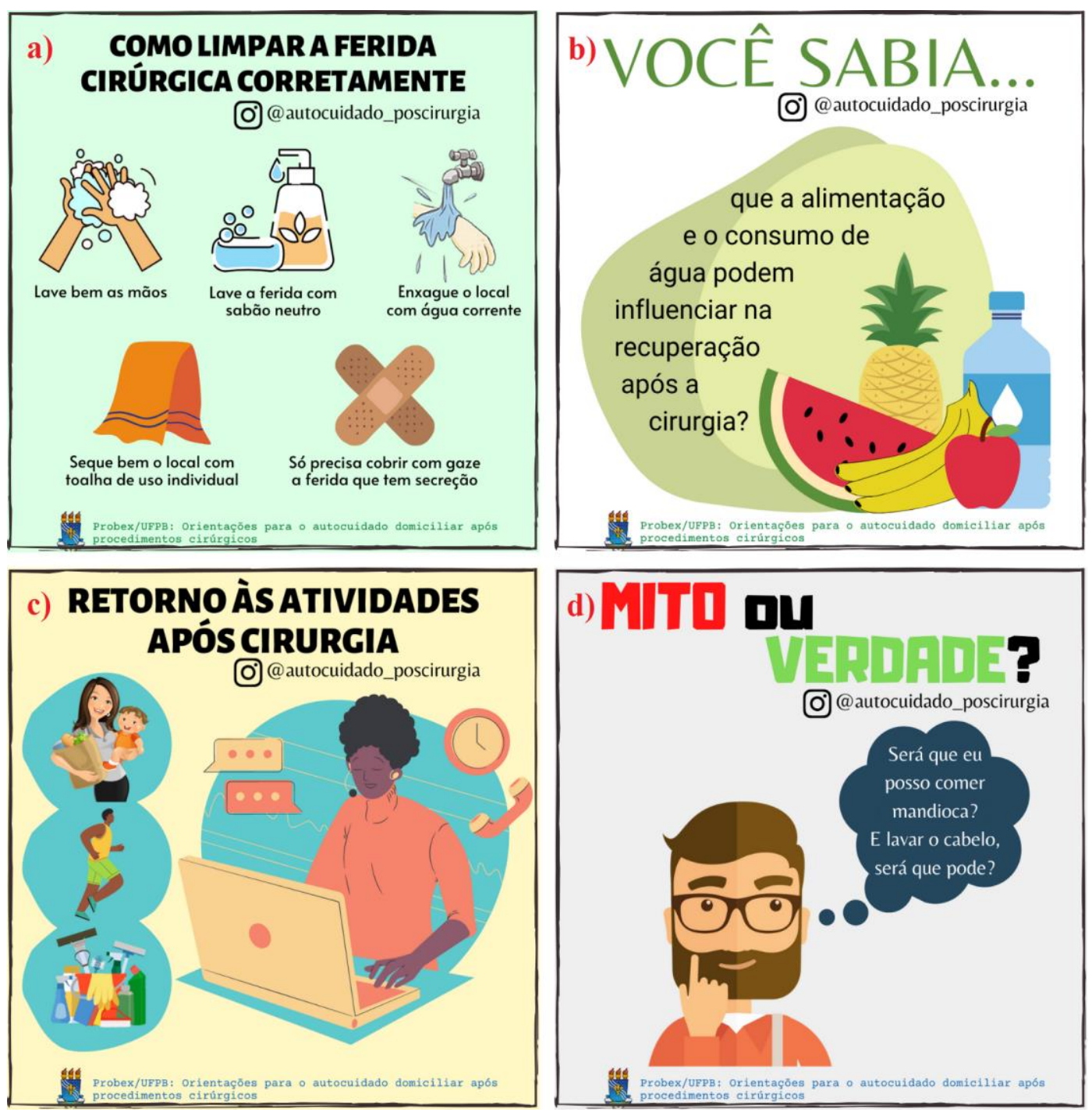

Figura 1. Artes das publicações sobre cuidados gerais no pós-operatórios

$\mathrm{Na}$ sequência, as publicações passaram a ocorrer três vezes por semana (nas terças-feiras, quintas-feiras e sábados), sendo abordada uma cirurgia por semana, contemplando indicações terapêuticas e recomendações para o autocuidado domiciliar a partir de séries temáticas ("conceito e indicações" nas terças, "orientações específicas" nas quintas, e "mito ou verdade" nos sábados).

Em relação às séries temáticas publicadas semanalmente, utilizamos uma imagem para cada tipo de cirurgia acompanhada de legenda explicando o tema, na qual será postada três vezes na semana com os seguintes tópicos: 1) "conceito e indicação" na qual está sendo abordado o tipo de cirurgia e em quais situações esse tipo de procedimento é indicado; 2) "orientações específicas", na qual vem abordando os cuidados específicos com a cirurgia que são necessários para o autocuidado domiciliar, além daquelas que já foram descritas nas orientações gerais e 3) "mito ou verdade" que tem como objetivo desvendar os saberes populares de uma forma bem dinâmica, classificando como mito ou verdade. Então, para cada cirurgia, uma nova imagem é criada, a qual será publicada três vezes na semana, seguindo a série dos conteúdos. 


\section{Apresentação da página}

O perfil do projeto no Instagram, possui uma página inicial que é visualizada pelos seguidores, essa página é composta por alguns itens que favorece a disseminação do conteúdo e a interação com o seguidor, tais como: 1) uma foto com o nome do projeto, número de publicações, número de seguidores e quantos seguidores o perfil está seguindo, 2) biografia, 3) opção para seguir a página, enviar mensagem e acessar o email, 4) destaques com os stories salvos, 5) vídeos IGTV e 6) publicações no Feed (Figura 2).

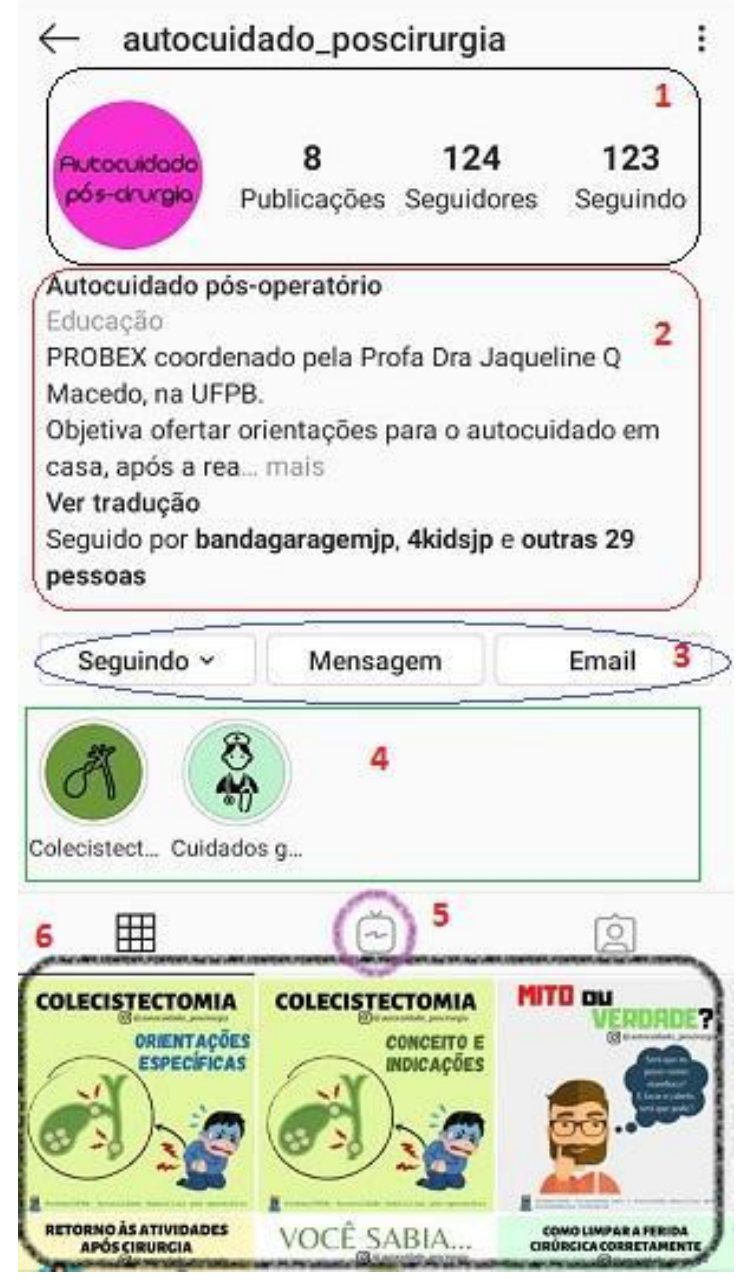

Figura 2. Imagem da página inicial do perfil no Instagram

No que diz respeito a biografia, é um campo onde pode preencher informações relevantes para o seu público, um site, caso tenha, telefone, endereço da sua empresa, uma chamada para uma ação específica, etc. É sua identidade, pelo qual pode descrever de maneira que seu público compreenda qual é o serviço ou conteúdo oferecido [Luca e Galeazzi 2019].

Importante considerar que, ao utilizar este perfil, o contato com os usuários torna-se contínuo, de modo que tanto por meio dos comentários como por meio do direct, faz-se possível interagir, solucionar dúvidas e aprofundar orientações sobre as temáticas abordadas neste projeto de extensão. 
O uso das TICs, principalmente o Instagram, vem se destacando como uma excelente ferramenta para promover a educação em saúde, pois permite a disseminação do conhecimento através de postagens interativas com imagens e textos explicativos. Devido ao seu alto potencial de interatividade, tendo como características o compartilhamento de informações e a construção coletiva do conhecimento, desempenha o papel de mediador entre a informação científica e a população leiga, traduzindo-a para uma linguagem mais acessível e contextualizando-a com o cotidiano da sociedade [Almeida e Stasiak 2013, Melo, Fonseca e Vasconcelos-Silva 2017].

\section{Considerações finais}

O uso das TICs e das mídias sociais está modificando o modo como a saúde interage com os usuários da saúde. Com essa prática da educação em saúde, utilizando ferramentas virtuais gratuitas e públicas, as ações do projeto assumem uma abrangência para toda a sociedade, tornando o conhecimento disponível para todos que tenham o acesso e o interesse de conhecer.

Sendo o Instagram uma ferramenta que permite a publicação de fotos e vídeos e uma interação social entre as pessoas, permite a aquisição do objetivo do projeto em promover a educação em saúde sobre o autocuidado domiciliar após cirurgias; assim como atende amplamente às metas de popularização do conhecimento científico, como Projeto de Extensão. E para além disso, percebe-se como o uso da tecnologia de informação e comunicação pode aproximar a área da saúde (profissionais, serviços, academia) da sociedade.

\section{Referências}

Aguiar, A. C. L., Guimarães, J. M. X., Ferreira, H. S., Almeida, K. T. C., Ribeiro, T. F. S., Anchieta, T. M., Carneiro, M. S. S. e Silva, B. C. (2018). "Blog como ferramenta educacional: contribuições para o processo interdisciplinar de educação em saúde", Reciis: Rev Eletron Comun Inf Inov Saúde, v.12, n.2, p.220-31.

Almeida, M. e Stasiak, D. (2013). “A promoção da saúde nas mídias sociais: uma análise do perfil do Ministério da Saúde no Twitter", In: Anais XV Congresso de Ciências da Comunicação na Região Centro-Oeste, Rio Verde, Brasil, Intercom.

Baller, S., Dutta, S. e Lanvin, B. (2016), The Global Information Technology Report 2016: Innovating in the Digital Economy, World Economic Forum, $1^{\text {st }}$ edition.

Bissolotti, K., Nogueira, H. e Pereira, A. (2014). "Potencialidades das mídias sociais e da gamificação na educação a distância", CINTED: Novas Tecnologias na Educação, v.12, n.2.

Boulos, M. N. K., Giustini, D. M. e Wheeler, S. (2016). "Instagram and WhatsApp in Health and Healthcare: An Overview", Future Internet, v.8, n.3, p.37.

Chaves, A. S. C. et al (2018). "Uso de aplicativos para dispositivos móveis no processo de educação em saúde: reflexos da contemporaneidade", Revista Humanidades e Inovação, v.5, n. 6, p. 34-42. 
Colnago, C. K. (2015) "Mídias e redes sociais digitais: conceitos e práticas", In: Estratégias de comunicação nas mídias sociais, Edited by Wilson da Costa Bueno, São Paulo, Brasil, Manole, p. 3-22.

Costa, D. O., Araújo, F. A., Xavier, A. S. G., Araújo, L. S., Silva, U. B., Santos, E. A. e Ferreira, S. L. (2018). "Autocuidado de homens com priapismo e doença falciforme", Rev. Bras. Enferm., v.71, n.5, p. 2418-2424.

Dalmolin, A. et al. (2016). "Vídeo educativo como recurso para educação em saúde a pessoas com colostomia e familiares", Revista gaúcha de Enfermagem, v.37, e68373.

Freitas, C. E. P., Borges, M. V., Rios, J. R. A. C. (2016). “O algoritmo classificatório no feed do Instagram”, In: Anais $39^{\circ}$ Congresso Brasileiro de Ciência da Comunicação, São Paulo, Brasil, Intercom.

Luca, D. e Galeazzi, T. L. (2019). "O Instagram como estratégia de marketing digital na empresa nutriativa", Trabalho de Conclusão de Curso: Técnico, Instituto Federal de Santa Catarina, Brasil.

Massara, C. L, et al (2016). "Caracterização de materiais educativos impressos sobre esquistossomose, utilizados para educação em saúde em áreas endêmicas no Brasil”, Epidemiol. Serv. Saúde, v.25, n.3, p.575-584.

Melo, A. S. L., Santos, A. A., Braga, A. G. S. e Silva, C. P. (2020). "Utilização das mídias sociais para educação em saúde pela LAPFITO: do instagram a oficinas de saúde e a interação entre academia e comunidade", In: Anais $4^{\circ}$ Seminário Tecnologias Aplicadas à Educação e Saúde, Salvador, Brasil, UNEB, p. 207-214.

Melo, M. C., Fonseca, C. M. F. e Vasconcellos-Silva, P. R. (2017). "Internet e mídias sociais na educação em saúde: o cenário oncológico", Cadernos do Tempo Presente, n. 27 , p. $69-83$.

Neto, N. M. G., Áfio, A. C. E., Leite, S. S., Silva, M. G., Pagliuca, L. M. F. e Caetano, J. Á. (2019). "Tecnologias para educação em saúde de surdos: revisão integrativa", Texto Contexto Enferm, v.28, e20180221.

Oliveira, F. A. et al (2018). "Atividades de educação em saúde realizadas com grupo de idosas para promoção do autocuidado em saúde", Extensio: R. Eletr. de Extensão, v.15, n.28, p. 137-150.

Porto, C. (2018) "Porque ignorar o algoritmo do Instagram pode prejudicar seu engajamento", https://www.camilaporto.com.br/instagram/algoritmo-do-instagram/, Maio.

Queirós, P. J. P., Vidinha, T. S. S. e Filho, A. J. Almeida (2014). “Autocuidado: o contributo teórico de Orem para a disciplina e profissão de Enfermagem", Revista de Enfermagem Referência, Série IV, n.3, p.157-164.

Sá, K. M. et al (2018). "Mídias Sociais como ferramenta de apoio às práticas integrativas em saúde na área de plantas medicinais", Vittalle, v.30, n.1, p. 144-1581.

Santos, F. D. R. P., Nunes, S. F. L., Pascoal, L. M., Silva, J. O. e Almeida, R. P. (2015). "Educação em saúde para pacientes no pós-operatório de cirurgias torácicas e abdominais", Rev. Ciênc. Ext., v.11, n.1, p.171-177. 
Santos, J. C. (2018). "Comparação das estratégias educativas em diabetes Mellitus em grupo e visita domiciliar", Dissertação de mestrado, Escola de Enfermagem da Universidade Federal de Minas Gerais, Brasil.

Sarwer-Foner, S. N. D. (2018). "Impacto das mídias sociais na mudança de hábitos de higiene bucal em escolares", Trabalho de Conclusão de Curso: graduação em Odontologia, Universidade Federal de Santa Catarina, Brasil.

Souza, D. A. S., Reis, I. A., Cortez, D. N., Afonso, G. S. e Torres, H. C. (2017). "Avaliação da visita domiciliar para o empoderamento do autocuidado em diabetes", Acta Paul Enferm., v.30, n.4, p.350-7.

Tavares, A. P. C., Leite, B. S., Silveira, I. A., Santos, T. D., Brito, W. A. P. e Camacho, A. C. L. F. (2018). "Análise das publicações nacionais sobre educação a distância em enfermagem: revisão integrativa”, Rev. Bras. Enferm, v.71, n.1, p.227-36.

Thomas, L. S., Fontana, R. T. (2019). "O instagram como ferramenta para educação em saúde", In: Anais $6^{\circ}$ Congresso Internacional em Saúde, Rio Grande do Sul, Brasil, UNIJUI.

Vieira, F. S. et al (2017). "Inter-relação das ações de educação em saúde no contexto da Estratégia Saúde da Família: percepções do enfermeiro", J. res.: fundam. care. online, v.9, n.4, p.1139-1144. 